multivariate analysis on the ESGO/ESMO/ESTRO risk class classification and results were statistically significant for both DFS $(p=0,003)$ and OS $(p=0,0001)$.

Conclusion Almost all the considered prognostic factors influence the presence of recurrence, but the stage is the most important factor while LVSI correlates with distance metastasis. The definition of the risk factors must be considered to develop targeted therapeutic pathways.

Disclosures The authors declare that the research was conducted in the absence of any commercial or financial relationship that could be construed as a potential conflict of interest.

\section{METFORMIN AS A PREVENTIVE AND THERAPEUTIC MODALITY IN ENDOMETRIAL CANCER: A SYSTEMATIC REVIEW AND META-ANALYSIS OF RANDOMIZED CONTROL TRIALS}

Anastasia Prodromidou, Sofia Lekka, Alexandros Fotiou, Victoria Psomiadou, Dimitrios Giannoulopoulos, Christos R lavazzo. Metaxa Memorial Cancer Hospital; Metaxa Memorial Cancer Hospital of Piraeus; Gynecologic Oncolog

\subsection{6/ijgc-2020-ESG0.78}

Introduction/Background Endometrial cancer (EC) is the most commonly diagnosed gynecological malignancy in the developed countries. Obesity, diabetes mellitus and infertility are some of the contributory factors. Some patients with EC wish to preserve their fertility or others have several comorbidities that contraindicate surgery. These groups of patients could benefit from a conservative treatment strategy such as the use of metformin. This agent is an option in women with increased EC risk as well as in those with atypical endometrial hyperplasia.

Methodology We evaluated the protective effects of metformin in EC patients, its preventive role in breast cancer and obese patients and its effectiveness, safety and efficacy in addition to progesterone monotherapy in treatment of fertility sparing candidates. We reviewed the literature and then conducted a meta-analysis of the relevant parameters. A total of 6 studies was included in the meta-analysis.

Results Comparing the pre-surgical treatment with metformin versus placebo, meta-analysis of mean difference in Ki-67 after treatment among two groups, revealed no difference (MD 7.10, 95\% CI -23.31 to 9.11, p=0.39). Meta-analysis of fertility sparing EC management with a combination of megestrol acetate (MA) and metformin (500 $\mathrm{mg}$ three times a day) in comparison with monotherapy with $160 \mathrm{mg}$ daily MA revealed no difference in either complete response or partial response rates (166 patients OR 2.94, 95\% CI 0.85 to 10.15 , $\mathrm{p}=0.09$ and 166 patients OR $0.76,95 \%$ CI 0.34 to 1.66 , $\mathrm{p}=0.49$, respectively). Regarding breast cancer survivors under tamoxifen, metformin was related with significantly reduced median endometrial thickness after 52 weeks of evaluation when compared to women in placebo group $(2.3 \mathrm{~mm}$ vs 3.0 $\mathrm{mm}, \mathrm{p}=0.05)$.

Conclusion Metformin neither was found to have a preventative role against the development of endometrial cancer nor a beneficial one in addition to the progesterone monotherapy for EC fertility sparing candidates. However, metformin was found to be protective in breast cancer survivors under tamoxifen.

Disclosures Nothing to disclose.

\section{9} COMBITEC: MULTICENTRIC RETROSPECTIVE STUDY ON SENTINEL LYMPH NODE DETECTION BY COMBINED ICG + 99MTC VERSUS EXCLUSIVE ICG IN ENDOMETRIAL CANCER

${ }^{1}$ Vicente Bebia Conesa, ${ }^{2}$ Marc Barahona, ${ }^{3}$ Cristina Almansa, ${ }^{4}$ Pablo Padilla Iserte, ${ }^{2}$ Lola Marti, ${ }^{2}$ Ponce Sebastiá Ponce, ${ }^{3}$ Alvaro Tejerizo, ${ }^{4}$ Santiago Domingo, ${ }^{5}$ Antonio GilMoreno, ${ }^{6}$ Silvia Cabrera Diaz. ${ }^{1}$ Hospital Universitari Vall D'hebron; Gynecologic Oncology; ${ }^{2}$ Hospital Universitari de Bellvitge; Gynecologic Oncology; ${ }^{3}$ Hospital Universitario 12 de Octubre; Gynecology; ${ }^{4}$ Hospital Universitari La Fe; Gynecologic Oncology; ${ }^{5}$ Fundació Vall Hebron; Institut de Recerca; Gynecologic Oncology; ${ }^{6}$ Hospital Universitari Vall D'hebron; Gynecology; Gynecologic Oncology

\subsection{6/ijgc-2020-ESG0.79}

Introduction/Background Despite its extended use, there is scarce evidence about the combined use of $99 \mathrm{mTc}$-albumin nanocolloid $(99 \mathrm{mTc})$ and indocyanine green (ICG) for the detection of sentinel lymph node (SLN) in endometrial cancer, when compared to ICG alone. The aim of this study is to compare the detection parameters of both methods.

Methodology Multicentric retrospective study (November 2015-June 2020) including patients diagnosed with endometrial atypical hyperplasia or initial preoperative stage endometrial carcinoma (FIGO I-II) who underwent SLN biopsy by cervical injection of: a) ICG intraoperatively, or b) $99 \mathrm{mTc}$ preoperatively, and ICG intraoperatively (ICG $+99 \mathrm{mTc})$.

Results A total of 180 patients were included, 51\% ( $\mathrm{n}=92)$ in the ICG group and $49 \%(n=88)$ in the ICG $+99 m$ Tc group. $86.7 \%$ of the patients presented endometrioid histology, and $58.7 \%$ were preoperatively classified as low risk, according to the ESMO/ESGO/ESTRO criteria. The vast majority of the procedures (99.4\%) were performed by a minimally invasive approach. Both groups were comparable regarding their basal characteristics, except for a higher body mass index (27.6 vs. $30.3 \mathrm{~kg} / \mathrm{m} 2, \mathrm{p}=0.014)$ in the ICG $+99 \mathrm{mTc}$ group and a bigger proportion of robotic-assisted procedures $(54.4$ vs $29.6 \%$, $\mathrm{p}=0.001)$ in the ICG group.

Global detection rate was 92.8\% (IC 95\%: 88.0-95.7), without statistically significant differences among groups (ICG:94.6\% vs ICG+99mTc:90.9\%, p=0.344). No significant differences were observed in the pelvic bilateral mapping rate $(71.6 \%$, ICG: $70.7 \%$ vs ICG $+99 \mathrm{mTc}: 71.6 \%, \mathrm{p}=0.890)$ or the aortic mapping rate $(5.6 \%$, ICG: $8.7 \%$ vs ICG $+99 \mathrm{mTc}: 2.3 \%, \mathrm{p}=0.058)$.

When ICG $+99 \mathrm{mTc}$ was used, surgical procedures were 30 minutes longer when compared to ICG $(150$ vs $180 \mathrm{~min}$, $\mathrm{p}=0.003)$. In 12 patients $(6.7 \%)$ at least one positive SLN was found (ICG:9.8\% vs ICG+99mTc:3.4\%, p=0.164).

No significant differences were observed regarding the empty node packets rate or the number of SLNs retrieved per patient. There were no patients with a positive lymphadenectomy specimen and a negative SLN, thus sensitivity was $100 \%$.

Conclusion Combining preoperative $99 \mathrm{mTc}$ to intraoperative ICG did not improve SLN detection in endometrial cancer, but resulted in longer procedures.

Disclosures Nothing to disclose.

\section{PREDICTIVE ROLE OF RADIOMIC FOR POST-OPERATIVE COMPLICATIONS OF LYMPHADENECTOMY IN EC PATIENTS}

${ }^{1}$ Francesco Magni, ${ }^{2}$ Massimiliano Fambrini, ${ }^{2}$ rene Paternò, ${ }^{2}$ Virginia Taddei, ${ }^{2}$ Flavia Sorbi, ${ }^{2}$ Silvia Lucarini, ${ }^{2}$ Mazzoni Laura, ${ }^{2}$ Felice Petraglia. ${ }^{2}$ Careggi University Hospital; Gynecology and Obstetrics; ${ }^{2}$ Careggi University Hospital

10.1136/ijgc-2020-ESGO.80 